\title{
Acumulação de matéria seca pelo cafeeiro conilon ${ }^{1}$
}

\author{
Scheilla Marina Bragança ${ }^{2}$, Herminia Emilia Prieto Martinez', Helio Garcia Leite ${ }^{4}$, Lucio Pereira Santos ${ }^{5}, J_{o s e ́}$ \\ Antonio Lani ${ }^{6}$, Carlos Sigueyuki Sediyama, Víctor Hugo Alvarez $V^{7}$
}

\section{RESUMO}

Com o objetivo de caracterizar a acumulação de matéria seca pelo cafeeiro conilon (Coffea canephora Pierre), um experimento foi conduzido em Marilândia/ES, no delineamento de blocos casualizados, com 24 tratamentos (época de amostragem) e três repetições. Utilizou-se a variedade clonal EMCAPA 8111, plantada em outubro de 1995. Constatouse que o cafeeiro conilon apresentou padrão sigmoidal de crescimento até os seis anos de idade, com acumulação crescente de massa de matéria seca. Houve declínio na taxa de crescimento absoluto após 48 meses de idade e diminuição na taxa de crescimento relativo após o primeiro mês de idade.

Palavras-chave: Análise de crescimento, Coffea canephora, partição, taxa de crescimento absoluto, taxa de crescimento relativo.

\begin{abstract}
\section{Dry matter accumulation by Conilon coffee}

The objective of this study was to characterize the dry matter accumulation of Conilon (Coffea canephora Pierre) coffee plants. The experiment was conducted in Marilândia/ES, Brazil, in an orchard with the clone 02, from the clonal variety EMCAPA 8111, established in October 1995. The experiment was arranged in a randomized block design, with twenty-four treatments (date of sampling) and three repetitions. It was found that up to six years of cultivation, plants of Conilon coffee show a sigmoid growth pattern, with a continuous increase in dry matter accumulation. There was a decline in the absolute growth rate after 48 months of cultivation, whereas for the relative growth rate, the reductions were observed after only one month of cultivation.
\end{abstract}

Key words: Growth analysis, absolute growth rate, Coffea canephora, partition, relative growth rate.

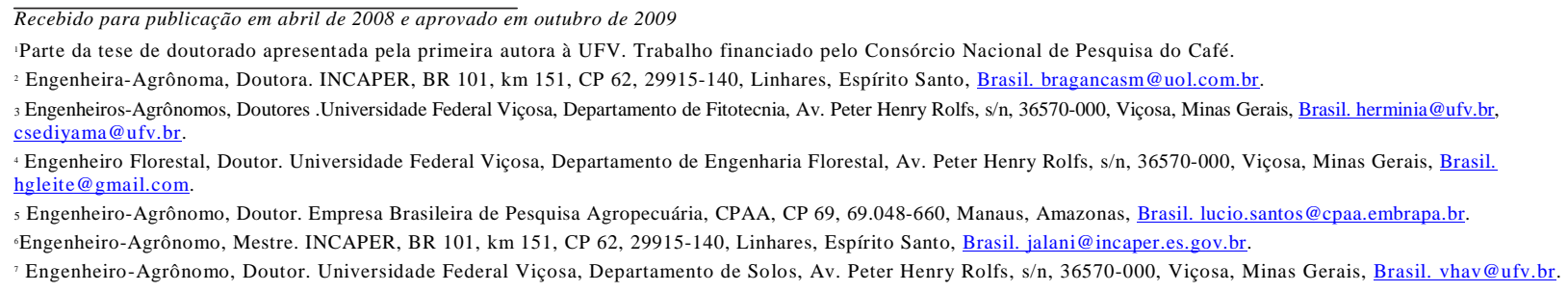

Rev. Ceres, Viçosa, v. 57, n.1, p. 048-052, jan/fev, 2010 


\section{INTRODUÇÃO}

Os estudos sobre análise de crescimento possibilitam acompanhar o desenvolvimento das plantas como um todo e a contribuição dos diferentes órgãos no crescimento total, permitindo conhecer o seu funcionamento e suas estruturas. É, também, um instrumento que tem sido usado com o objetivo primordial de gerar descrição clara do padrão de crescimento da planta ou de partes dela, permitindo comparações entre situações distintas, podendo ser aplicada às mais diversas modalidades de estudos (Liedgens, 1993).

De modo geral, as plantas lenhosas apresentam aumento inicial de peso de massa seca de órgãos e/ou tecidos caracterizados por modelos lineares (Kozlowski \& Pallardy, 1996). Contudo, vários mecanismos internos de controle induzem a uma modificação dessa relação que pode, em um período maior, ser descrita de forma mais adequada por uma curva sigmoidal.

Vários aspectos do crescimento do Coffea arabica têm sido estudados, principalmente no Brasil, sendo o acúmulo de matéria seca em função do tempo um deles. Estas pesquisas tomaram impulso na d6cada de $1950 \mathrm{com}$ os trabalhos de Catani \& Moraes (1958), que estudaram a variedade Bourbon Vermelho até os cinco anos de idade. Estes autores observaram que esta variedade apresentou um aumento crescente de matéria seca, alcançando um total de $10.174 \mathrm{~g} /$ planta ao final do experimento, distribuídos entre raiz (1.352 g/planta), tronco (3.398 g/planta), ramos (2.480 g/planta), folhas (1.263 g/planta) e frutos (1.681 g/planta).

Posteriormente, Catani et al. (1965), trabalhando com a variedade Mundo Novo (Coffea arabica), afirmaram que o cafeeiro de 10 anos de idade está em pleno crescimento. Os resultados obtidos neste trabalho mostraram que estavariedade acumulou uma matéria seca total de $19.417 \mathrm{~g} /$ planta ao final desse período. Em trabalho mais recente, Corrêa et al. (1985), trabalhando com as cultivares Catuaí Vermelho IAC 81 e Mundo Novo IAC 379/19, também concluíram que a quantidade de matéria seca total foi crescente até os 6,5 anos de idade nas duas variedades estudadas, que alcançaram um valor máximo de $24,6 \mathrm{~kg} / \mathrm{planta}$ e $27,69 \mathrm{~kg} /$ planta, respectivamente. Resultados semelhantes foram obtidos por Cietto et al. (199 1) com o cultivarCatuaí de cinco anos de idade.

Assim, objetivou-se com este trabalho determinar o acúmulo de matéria seca e a taxa de crescimento do cafeeiro Conilon (Coffea canephora Pierre).

\section{MATERIAL E MÉTODOS}

O experimento foi instalado em outubro de 1995, na Fazenda Experimental do INCAPER, em Marilândia-ES, a uma altitude de $150 \mathrm{~m}$, latitude de $19^{\circ} 24^{\prime} 31^{\prime \prime}$ sul e longitu de $40^{\circ} 31^{\prime} 24^{\prime \prime}$ oeste, sobre Latossolo Vermelho Amarelo.
O município é caracterizado pelo período de novembroa fevereiro úmidos; março, abril e outubro parcialmente úmido; e maio a setembro seco. A média anual das temperaturas máximas é de $33,5^{\circ} \mathrm{C}$, e das mínimas, de $13,9^{\circ} \mathrm{C}$ (Feitosa et al., 1999).

De acordo com a análise do solo, foram aplicados 30 g/cova de $\mathrm{P}_{2} \mathrm{O}_{5}$ e $20 \mathrm{~g} /$ cova de FTE BR 12 misturados na cova de plantio $\left(40\right.$ x 40 x $40 \mathrm{~cm}$ ) e $20 \mathrm{~g} /$ cova de $\mathrm{Ne} \mathrm{K}_{2} \mathrm{O}$ em cobertura. Empregou-se calcário dolomítico em área total e na cova, cujas doses foram calculadas pelo m6todo da saturação em bases, usando-se $70 \%$ como valor de referência. No período de formação das plantas foram utilizadas $50 \mathrm{~g} /$ cova de $\mathrm{N}$ e $40 \mathrm{~g} /$ cova de $\mathrm{K}_{2} \mathrm{O}$, respectivamente. $\mathrm{Na}$ fase de produção, elas foram conduzidas com $500 \mathrm{~kg}$ ha $^{-1} \mathrm{~N}, 75 \mathrm{~kg}$ ha- $1 \mathrm{P}_{2} \mathrm{O}_{5}$ e $450 \mathrm{~kg} \mathrm{ha}^{-1} \mathrm{~K}_{2} \mathrm{O}$, em três parcelamentos, conforme Bragança et al. (2001b). No experimento, as plantas foram conduzidas sem poda até o sexto ano de idade.

Utilizou-se o delineamento experimental de blocos casualizados com 24 tratamentos (época de amostragem) e três repetições. As amostragens foram trimestrais a partir do transplantio. Cada bloco constou de 100 plantas úteis da variedade clonal EMCAPA 8111 (Braganga et al.,2001 a), conduzidas no espaçamento 3,0 x 1,5 m, e cadaparcela de uma única planta útil circundada pelos outros clones da variedade, que constituíram a bordadura. Em cada época de amostragem tomou-se aleatoriamente umaplanta competitiva, cujas vizinhas não tivessem sido removidas em amostragens anteriores. As plantas foramcoletadas inteiras, incluindo-se as raízes. Para isto, foramabertas trincheiras, com enxadão, ao seu redor, sendo aremoção completada com jatos d'água.

Em cada parcela experimental foram determinadas as seguintes variáveis: massa de matéria seca total (MSTOTAL), massa de matéria seca de folhas (MSFO), massa de matéria seca do tronco + ramos ortotrópicos (MSTRRO), massa de matéria seca dos ramos plagiotrópicos (MSRP), massa de matéria seca do tronco + total de ramos (MSTRTORA) e massa de matéria seca das raízes (MSRA). Os frutos foram avaliados com casca e por diferença em relação à massa da matéria seca total. Flores e botões florais não foram considerados. As amostras foram lavadas e secadas em estufa com circulação forçada de ar a $70{ }^{\circ} \mathrm{C}$, até peso constante. Posteriormente, procedeu-se à pesagem da massa de matéria seca.

O modelo logístico foi ajustado para cada uma dessas variáveis, sendo sua relação funcional definida por $Y=\alpha\left(1+\beta e^{-\lambda t}\right)^{-1}+\varepsilon$, em que $Y$ é a variável dependente, $t 6$ a idade em meses, ${ }_{a, a} a$ são os parâmetros do modelo e é o erro aleatório. Conforme Drapper \& Smith (1980) e Fayad (1998), as taxas de crescimentos mensal, 
absoluto (AGR) e relativo (RGR) foram obtidas pela derivada primeira da equação de produção e pela relação AGR/ $\mathrm{Y}$, respectivamente, ou seja:

$$
\begin{aligned}
& A G R=\frac{d Y}{d t}=\frac{\hat{\gamma} \hat{Y}(\hat{\alpha}-\hat{Y})}{\hat{\alpha}} \mathrm{e} \\
& R G R=\frac{d Y / d t}{\hat{Y}}=\left(\frac{\hat{\gamma} \hat{Y}(\hat{\alpha}-\hat{Y})}{\hat{\alpha}}\right)\left(\frac{\hat{\alpha}}{1+\hat{\beta} e^{-\hat{t} t}}\right), \text { em que } \\
& \hat{Y}=\frac{\hat{\alpha}}{1+\hat{\beta} \cdot e^{-\hat{\gamma t}}}
\end{aligned}
$$

\section{RESULTADOS E DISCUSSÃO}

A produção total de massa de matéria seca foi crescente durante a condução do experimento, alcançando $15,94 \mathrm{~kg} /$ planta no $72^{\circ}$ mês (Figura 1). A massa da matéria seca das folhas, tronco + ramos ortotrópicos, ramos plagiotrópicos, raízes e tronco + total de ramos aumentou até alcançar 2,65 kg/planta, 7,39 kg/planta, 1,88 kg/planta, 2,69 kg/planta e 9,30 kg/planta, respectivamente (Figuras 2, 3, 4, 5 e 6). A massa de matéria seca dos frutos, calculada por diferença em relação à massa da matéria seca total, foi de $1,33 \mathrm{~kg} /$ planta. O modelo logístico ajustado para estas variáveis, com exceção dos frutos, descreveu o crescimento do café conilon de forma semelhante ao do padrão de crescimento de plantas lenhosas, descrito por Kozlowski \& Pallardy (1996).

Constatou-se, no $72^{\circ}$ mês, que a massa de matéria seca foi alocada principalmente no tronco + ramos ortotrópicos (46\%), seguindo-se folhas $(17 \%)$, raízes $(17 \%)$, ramos plagiotrópicos (12\%), e frutos (8\%). Estas informações, de acordo com Kozlowski \& Pallardy (1996), são importantes para a compreensão de como o ambiente e as práticas culturais interferem no crescimento, tendo Bartelink (1998) apresentado um modelo de partição de massa de matéria seca, em plantas lenhosas, com base no conceito de balanço estrutural. Embora existam conceitos teóricos de partição, eles incluem várias características que não podem ser quantificadas.

Diversas teorias têm sido propostas para descrever e/ou explicar a distribuição de assimilados e, consequentemente,

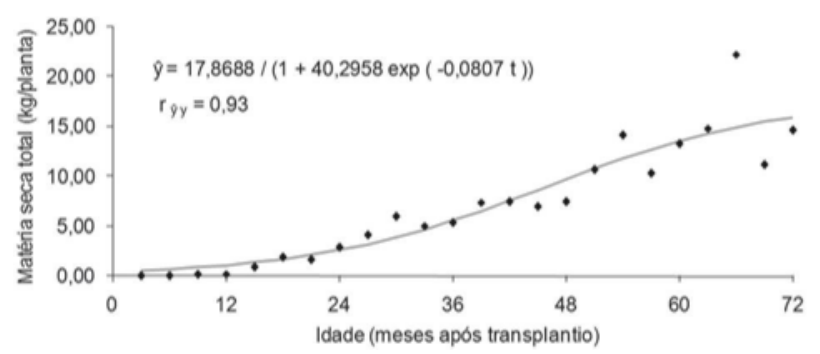

Figura 1. Massa de matéria seca total do cafeeiro conilon (Coffea canephora Pierre) do transplantio até 72 meses de idade. a partição da massa de matéria seca nos órgãos das plantas. Há a hipótese de que a distribuição de massa de matéria seca na planta seja regulada pela força do dreno dos órgãos, termo usado para descrever a habilidade competitiva de um órgão importar assimilados, quantificada pelas suas taxas potenciais de crescimento (Heuvelink, 1996).

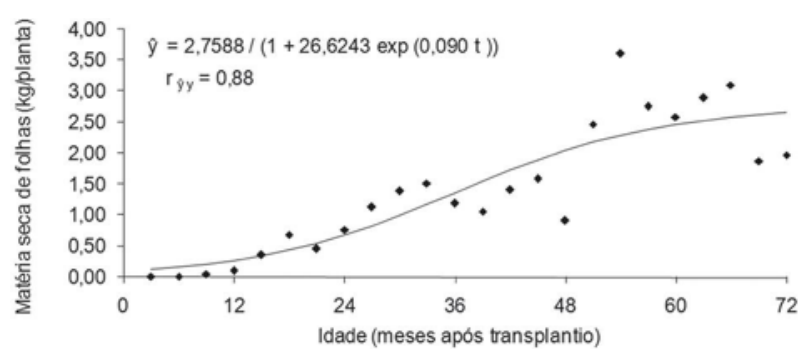

Figura 2. Massa de matéria seca das folhas do cafeeiro conilon (Coffea canephora Pierre) do transplantio até 72 meses de idade.

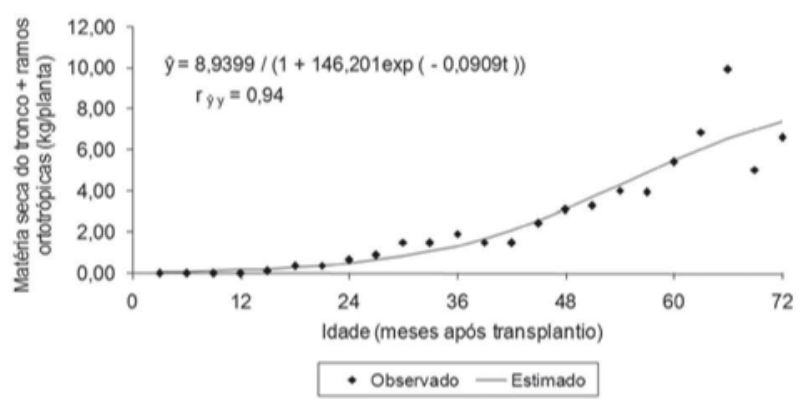

Figura 3. Massa de matéria seca do tronco + ramos ortotrópicos do cafeeiro conilon (Coffea canephora Pierre) do transplantio até 72 meses de idade.

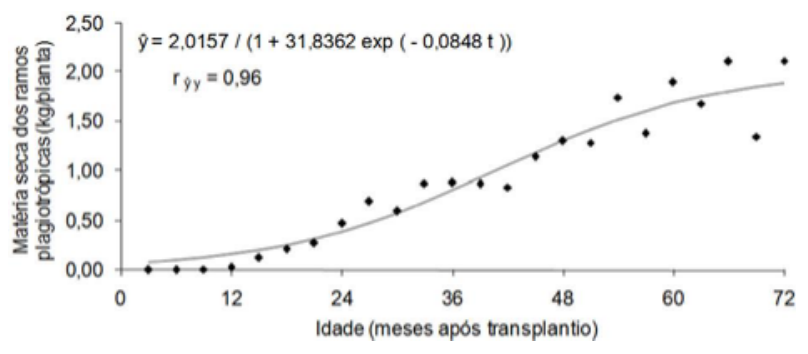

Figura 4. Massa de matéria seca dos ramos plagiotrópicos do cafeeiro conilon (Coffea canephora Pierre) do transplantio até 72 meses de idade.

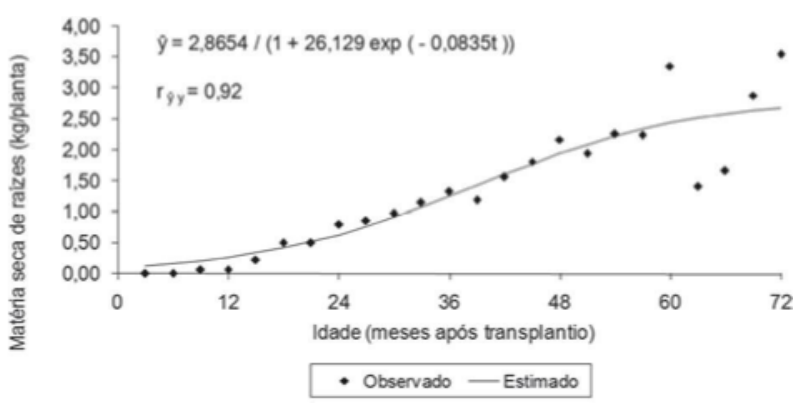

Figura 5. Massa de matéria seca de raízes do cafeeiro conilon (Coffea canephora Pierre) do transplantio até 72 meses de idade.

Rev. Ceres, Viçosa, v. 57, n.1, p. 048-052, jan/fev, 2010 


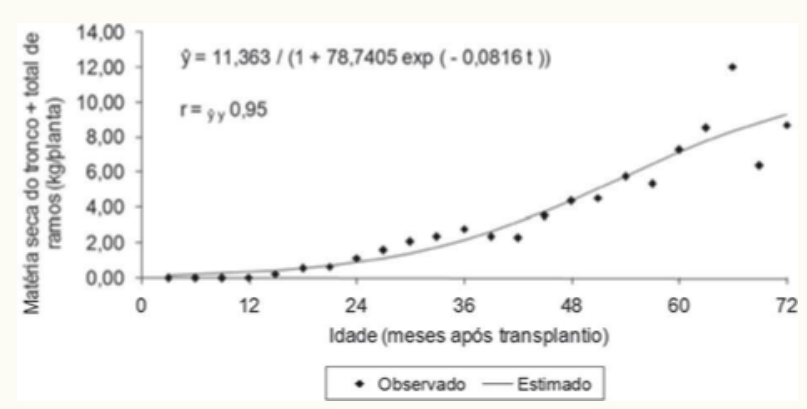

Figura 6. Massa de matéria seca do tronco + total de ramos do cafeeiro conilon (Coffea canephora Pierre) do transplantio até 72 meses de idade.

A taxa de crescimento absoluto do cafeeiro conilon aumentou de $0,04 \mathrm{~kg} / \mathrm{mês}$, no $1^{\circ}$ mês, para $0,36 \mathrm{~kg} / \mathrm{mês}$, no $48^{\circ}$ mês, diminuindo a seguir até alcançar $0,14 \mathrm{~kg} /$ mês, no $72^{\circ}$ mês. Por outro lado, a taxa de crescimento relativo, que depende fundamentalmente da área foliar útil para a fotossíntese e da taxa de fotossíntese liquida, diminuiu ao longo do ciclo de observações, variando de $0,08 \mathrm{~kg} \mathrm{~kg}^{-1}$ mês, no $2^{\circ}$ mês, a $0,01 \mathrm{~kg} \mathrm{~kg}^{-1}$ mês, no $72^{\circ}$ mês (Figura 7), indicando diminuição de massa de matéria seca por unidade de massa de matéria seca contida na planta no inicio do experimento. Diminuição nos valores da taxa de crescimento relativo, em função do tempo, é comum para algumas espécies, estando elas relacionadas aos decréscimos na taxa assimilatória liquida e na razão de área foliar.

Os resultados obtidos para as taxas de crescimento relativo de folhas, tronco + ramos ortotrópicos, ramos plagiotrópicos, tronco + total de ramos e raízes do cafeeiro conilon foram semelhantes àqueles obtidos quando se considerou a massa de matéria seca total; ou seja, houve diminuição de massa de matéria seca por unidade de massa de matéria seca contida na planta no inicio do experimento. Por outro lado, as taxas de crescimento absoluto aumentaram no transcorrer das avaliações, alcançando ovalor máximo entre o $36^{\circ}$ e o $57^{\circ}$ mês.

Cannell \& Kimeu (1971), comparando taxas de crescimento absoluto de cafeeiros Coffea arabica conduzidos com poda, no Kenya, com aqueles manejados sem poda,

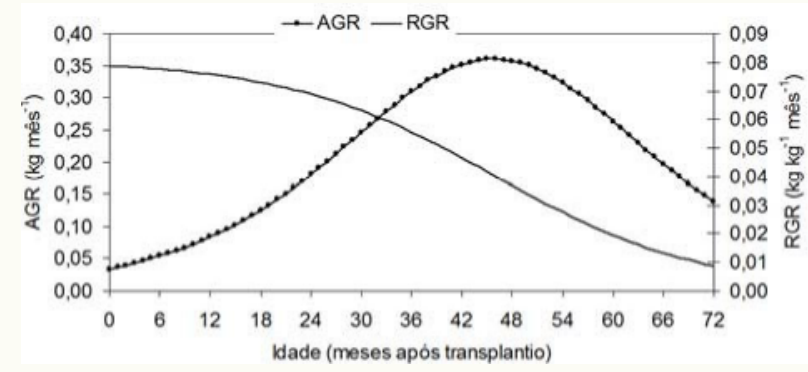

Figura 7. Taxas totais de crescimento absoluto e de crescimento relativo do cafeeiro conilon (Coffea canephora Pierre) do transplantio até 72 meses de idade.

em Campinas/SP (Brasil), mencionaram que os primeiros cresceram muito mais rapidamente (40 a $150 \mathrm{~g} / \mathrm{semana}$ ) do que os últimos (25 a $80 \mathrm{~g} / \mathrm{semana}$ ). Entretanto, como o crescimento do cafeeiro é influenciado por fatores relacionados à genética, ao clima e ao manejo da planta, dentre outros, estes autores citaram que os resultados referentes às taxas de crescimento absoluto devem ser aplicados com cautela. No presente trabalho, constatou-se que as AGRs apresentadas pelo cafeeiro conilon ( 360 g/mês), notadamente no $48^{\circ}$ mês, foram semelhantes àquelas encontradas por estes autores em cafeeiros conduzidos sem poda no Brasil.

Segundo Castro-Diez et al. (1998), as plantas diferem amplamente em suas RGRs, e essas diferenças podem ser atribuídas parcialmente às condições ambientais e características inerentes à espécie. Estes autores ressaltam que vários pesquisadores têm procurado identificar características da planta que expliquem essas variações, como aquelas relacionadas à fisiologia, anatomia foliar, partição de nutrientes, composição química e alocação de nutrientes.

Venkataramanan et al. (1983) estudaram as variações nas taxas de crescimento relativo de plântulas pertencentes a vários germoplasmas das espécies $C$. arabica e C. canephora durante dois períodos de crescimento $(4,5$ aos 7,5 meses e 7,5 aos 9,5 meses de idade). Observaram que as RGRs de plântulas com 9,5 meses de idade foram menores quando comparadas às daquelas com 7,5 meses, evidenciando diminuição em função do tempo. De modo geral, todos os cafés tipo arábica mostraram menores RGRs quando comparados à seleção 'S 274' de Robusta, que apresentou incremento de matéria seca por unidade de matéria seca presente de $0,1272 \mathrm{~g} \mathrm{~g}^{-1}$ semana e $0,1218 \mathrm{~g}^{\mathrm{g}-1}$ semana para o primeiro e segundo periodos estudados, respectivamente. Entretanto, os valores de RGRs encontrados para todos os germoplasmas de cafés estudados foram menores quando comparados a algumas espécies cultivadas como milho, feijão, canade-açúcar, algodão, beterraba-agucareira, batata, cevada, trigo e aveia. Alvim (1960) constatou valores muito mais altos de RGRs em cafeeiros da variedade Bourbon (C. arabica), o que pode ser atribuído a diferenças na idade e também às condições edafoclimáticas nas quais as plântulas foram cultivadas.

\section{CONCLUSÕES}

O cafeeiro conilon apresenta padrão sigmoidal de crescimento até os seis anos de idade, com acumulação crescente de massa de matéria seca. Há declínio na taxa de crescimento absoluto após 48 meses de idade e diminuição na taxa de crescimento relativo após o primeiro mês de idade. 


\section{REFERENCIAS}

Alvim PT (1960) Physiology of growth and flowering in coffee. Turrialba, 2:57-62.

Bartelink HH (1998) A model of dry matter partitioning in trees. Tree Physiology, 18:91-101.

Braganga SM, Carvalho CHS, Fonseca AFA, Ferrão RG (2001a) EMCAPA 8111, EMCAPA 8121, EMCAPA 8131: Variedades clonais de café Conilon para o Estado do Espírito Santo. Pesquisa Agropecuária Brasileira, 36:765-770.

Braganga SM, Lani JA \& Demuner LH (2001b) Café Conilon: Adubação e calagem. Vitória, INCAPER. 31p. (Circular Técnica 1).

Catani RA, Moraes FRP, (1958) A composição química do cafeeiro: Quantidade e distribuição de $\mathrm{N}, \mathrm{P}_{2} \mathrm{O}_{5}, \mathrm{CaO}$ e $\mathrm{MgO}$ em cafeeiro de 1 a 5 anos de idade. Revista de Agricultura, 33:45-52.

Catani RA, Pelegrino D, Bergamin Filho H, Glória NA \& Graner CAFA (1965) A absorgão de nitrogênio, fósforo, potássio, cálcio, magnésio e enxofre pelo cafeeiro. Coffea arabica L. variedade Mundo Novo aos dez anos de idade. Anais da Escola Superior de Agricultura "Luiz de Queiroz", 22:81-93.

Cannell MGR \& Kimeu BS (1971) Uptake and distribution of macronutrient in trees of Coffea arabica L. in Kenya as affected by seasonal climatic differences and the presence of fruits. Annals of Applied Biology, 68:213-230.

Castro-Díez P, Puyravaud JP \& Cornelissen JHC (1998) Stem anatomy and relative growth rate in seedlings of a wide range of woody plant species and types. Oecologia, 116:57-66.

Cietto S, Haag HP \& Dechen AR (1991) Acumulação de matéria seca e absorção de N, P e K pelo cafeeiro (Coffea arabica L. cv. Catuaí) com dois, três, quatro e cinco anos de idade, nas fases fenológicas de repouso, granação e maturação vegetando em um Latossolo Vermelho Amarelo, fase cerrado. Anais da Escola Superior de Agricultura "Luiz de Queiroz", 48:245-68.
Corrêa JB, Garcia AWR \& Costa PC (1985) Extragão de nutrientes pelo cafeeiro Mundo Novo e Catuaí. In: $12^{\circ}$ Congresso Brasileiro de Pesquisas Cafeeiras, Caxambú. Anais, IBC/GERCA. p.23-26.

Drapper NR \& Smith H (1980) Applied regression analysis, $2^{\text {nd }}$ ed. New York, J. Wiley. 709p.

Fayad JA (1998) Absorção de nutrientes, crescimento e produção do tomateiro cultivado em condições de campo e de estufa. Dissertagão de mestrado, Universidade Federal de Viçosa, Viçosa, $81 \mathrm{p}$.

Feitosa LR, Castro LLF, Resende M, Zangrande MB, Stocking MS, Borel RMA, Fullin EA, Cerqueira AF, Salgado JS, Feitoza HN, Stock LA, Mank AM, Dessaune Filho N, Mank AM, Feringa W \& Martinez JA (1999) Mapa das Unidades naturais do Espírito Santo. Vitória, EMCAPA, Mapa na escala 1:400 000. Colorido.

Heuvelink E (1996) Dry matter partitioning in tomato: validation of a dynamic simulation model. Annals of Botany, 77:71-80.

Kozlowski TT \& Pallardy SG (1996) Physiology of woody plants. 2nd ed. San Diego, Academic Press. 411p.

Liedgens MM (1993) Modelos numéricos para a descrigão do crescimento daplanta de soja (Glycine max L. Merril, cultivar IAC15) em condigões sazonais diferenciadas. Dissertagão de mestrado. Universidade Estadual de Campinas, Campinas, 101p.

Venkataramanan D, Vasudeva N, Raju KI, Ratageri MC \& Gopal NH (1983) The relative growth and net assimilation rate of certain cultivars of coffee. Journal of Coffee Research, 14:6-13. 